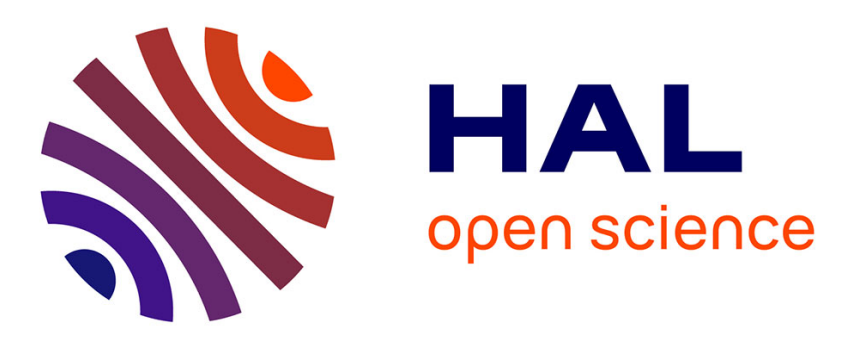

\title{
Structural Morphology and Performance of Plated Structures with Planar Quadrilateral Facets
}

\author{
Romain Mesnil, Olivier Baverel, Cyril Douthe, Jean-François Caron, Bruno
} Léger

\section{- To cite this version:}

Romain Mesnil, Olivier Baverel, Cyril Douthe, Jean-François Caron, Bruno Léger. Structural Morphology and Performance of Plated Structures with Planar Quadrilateral Facets. Journal of the International Association for Shell and Spatial Structures, 2017, 58 (1), 10.20898/j.iass.2017.191.845 . hal-01510086

\section{HAL Id: hal-01510086 \\ https://hal-enpc.archives-ouvertes.fr/hal-01510086}

Submitted on 26 Apr 2017

HAL is a multi-disciplinary open access archive for the deposit and dissemination of scientific research documents, whether they are published or not. The documents may come from teaching and research institutions in France or abroad, or from public or private research centers.

$$
\text { Copyright }
$$

L'archive ouverte pluridisciplinaire HAL, est destinée au dépôt et à la diffusion de documents scientifiques de niveau recherche, publiés ou non, émanant des établissements d'enseignement et de recherche français ou étrangers, des laboratoires publics ou privés. 


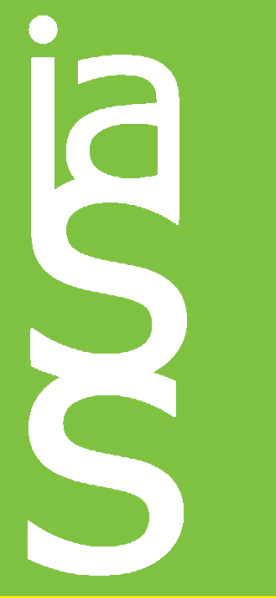

\section{JOURNAL OF}

THE INTERNATIONAL ASSOCIATION

\section{FOR SHELL AND SPATIAL}

\section{STRUCTURES}

Prof. D. h-C Eng .E. TORROJA, founder

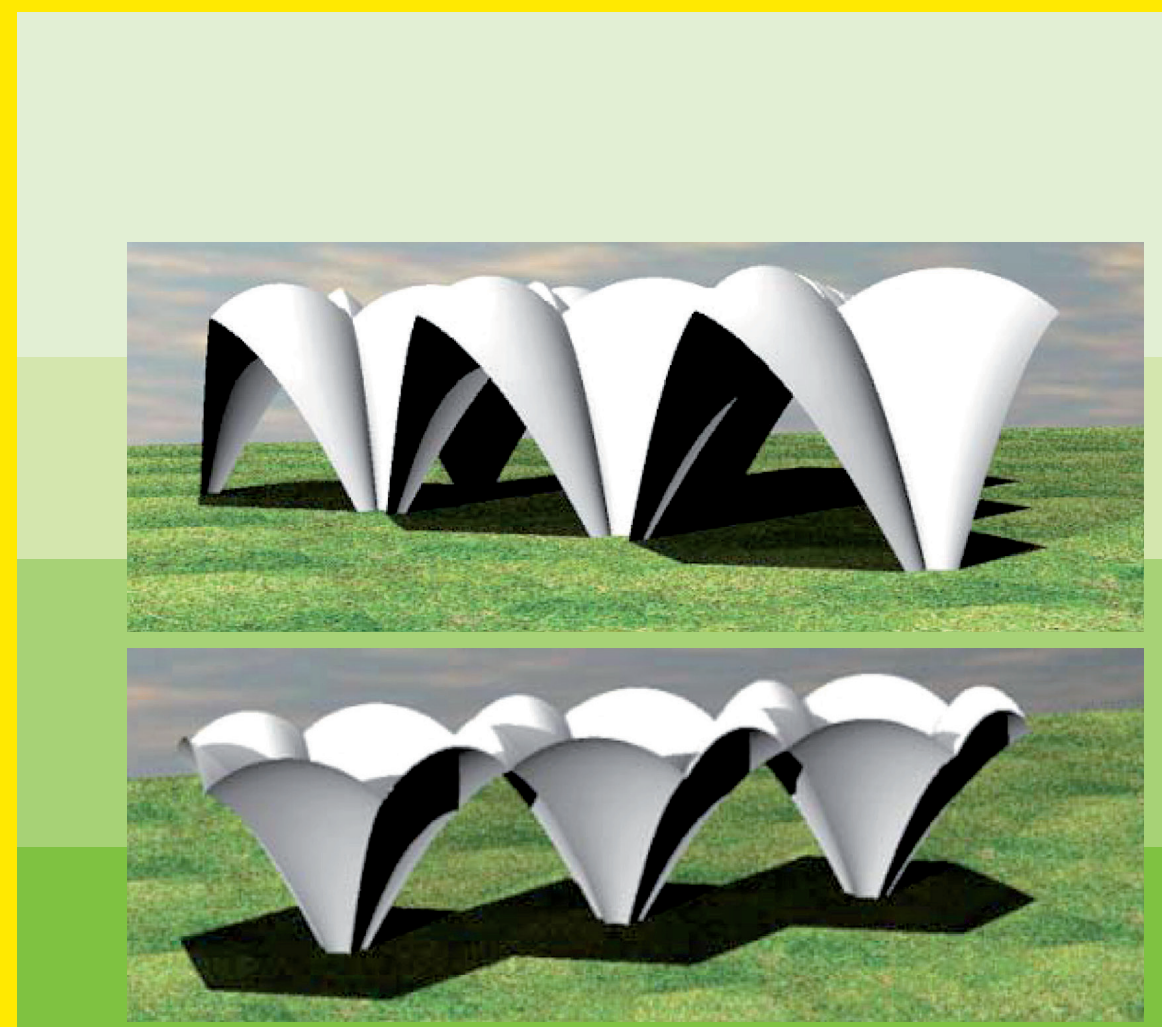

SPECIAL ISSUE NEW DIRECTIONS FOR SHELL STRUCTURES Guest Editors: S. ADRIAENSSENS and P. BLOCK Vol. 58 (2017) No. 1 March n. 191 ISSN: 1028-365X 


\section{Journa}

\section{VOL. 58 (2017) No. 1 \\ contents}

\section{n. 191 March}

\section{Memorial Statement}

Memorial to Ludevít Végh

N. Imagawa and T. Tarnai

Preface

Special Issue of WG5 on "New Directions for Shell Structures"

S. Adriaenssens and P. Block

Technical Papers

Structural Morphology and Performance of Plated Structures with Planar Quadrilateral Facets

R. Mesnil, O. Baverel, C. Douthe, J.-F. Caron and B. Leger

Structural Design of the Flexibly Formed, Mesh-Reinforced Concrete Sandwich

Shell Roof of NEST HiLo

D. Veenendaal, J. Bakker and P. Block

From a Weightless Bent Wire Coat Hanger to Shell Structures

Via the Beltrami Stress Tensor

S. Malek, A. McRobie, P. Shepherd and C. Williams

Structural Implementation of Slender Concrete Shells with Prefabricated Elements

P. Eisenbach and M. Grohmann

Hybrid Structural Skin: Prototype of a GFRP Elastic Gridshell Braced

by a Fiber-Reinforced Concrete Envelope

P. Cuvilliers, C. Douthe, L. du Peloux and R. Le Roy

Shells Made of Textile Reinforced Concrete - Applications in Germany

S. Scheerer, R. Chudoba, M.P. Garibaldi and M. Curbach

Design of Three Hypar Roofs Made of Guadua Bamboo

T. Michiels, L. Lu, R. Archer, S. Adriaenssens and G. Tresserra

Optimised High-Performance Concrete Shells for Parabolic Trough Collectors

C. Kämper, P. Forman, T. Stallmann, M.A. Ahrens, P. Mark and J. Schnell

Upcoming Events

COVER: Figure from paper by S. Scheerer, R. Chudoba, M.P. Garibaldi and M. Curbach

IASS Secretariat: CEDEX-Laboratorio Central de Estructuras y Materiales

Alfonso XII, 3; 28014 Madrid, Spain

Tel: 3491 3357491; Fax: 3491 3357422; iass@cedex.es; http://www.iass-structures.org

Printed by SODEGRAF ISSN:1028-365X Depósito legal: M. 1444-1960 


\title{
STRUCTURAL MORPHOLOGY AND PERFORMANCE OF PLATED STRUCTURES WITH PLANAR QUADRILATERAL FACETS
}

\author{
Romain MESNIL ${ }^{1,2}$, Olivier BAVEREL ${ }^{1}$, Cyril DOUTHE ${ }^{1}$, Jean-François CARON ${ }^{1}$ \\ and Bruno LEGER ${ }^{2}$
}

'Laboratoire Navier, UMR 8205, École des Ponts, CNRS, IFSTTAR, UPE, Champs-sur-Marne, France ${ }^{2}$ Bouygues Construction, France

Editor's Note: Manuscript submitted 20 September 2016; revision received 7 February 2017; accepted 16 February. This paper is open for written discussion, which should be submitted to the IASS Secretariat no later than September 2017.

DOI: https://doi.org/10.20898/j.iass.2017.191.845

\section{ABSTRACT}

The aim of this paper is to present some innovative strategies for the design of plated shell structures. Fabrication constraints are carefully considered, as the obtained shapes are covered with planar quadrilateral facets only. Different corrugation strategies are investigated, and our method guarantees extension of the shape generation to complex topologies. Few parameters control the amplitude of corrugations, which also gives us the opportunity to perform a sensitivity analysis on the influence of corrugations on the structural performance of folded-plate structures. The study focuses on the influence of the mechanical attachment between plates on the overall structural performance as well as fabrication rationality.

Keywords: Folded plate structures, free-form architecture, parametric design, fabrication-aware design, linear buckling analysis

\section{INTRODUCTION}

So-called folded plate structures have been used for decades (see an early patented example in [1]). These structures consist of rigid plate elements connected along the edges. Recently, numerical fabrication methods and more complex shape generation principles for folded plate structures extended their possibilities. Two of the main concerns of designers are structural efficiency and fabrication cost. The planarity of the plates is thus of particular importance in the structural morphology of folded plate structures.

This article proposes the use of a unifying framework for the generation of corrugated plated shell structures covered with planar quadrilaterals. The generated design space is thus constituted of constructible shapes: some illustrations of their formal possibilities are shown in Figure 1. The corrugation strategies introduced in this paper leave potential for structural optimisation.
The main contributions of the article follow:

- The use of the marionette method for the generation of PQ-meshes, previously introduced by the authors, and the introduction of two corrugation strategies.

- The assessment of the performance of corrugated plated shells with respect to different geometrical and mechanical parameters.

- A discussion on the convexity of the design space and recommendations for structural optimisation of folded plate structures.

- Design guidelines for folded plate structures. 

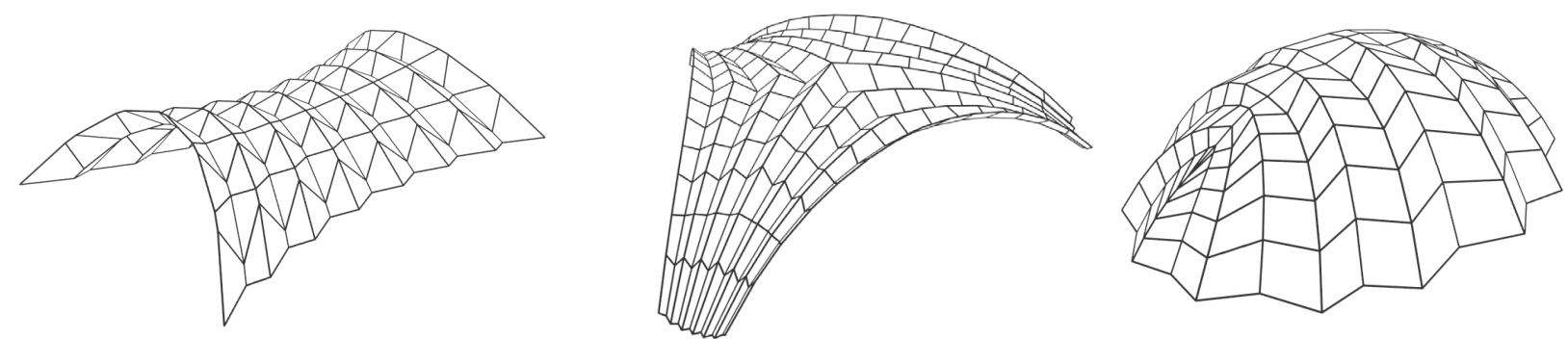

Figure 1: Several designs generated with the method presented in this paper, all facets are planar quadrilaterals. Two corrugation strategies are introduced in this article: the alternate translation (used in the left and middle) and the row translation strategy (used in the right)

\section{LITERATURE REVIEW}

Three approaches are used for the shape determination of plated structures. They approximate surfaces with planar panels, as it is a key element in the economy of the building envelope. The first strategy focuses on the study of origami, which benefits from a rich theoretical background. For structures that are not deployable, the use of a structure with potential mechanisms can however be questioned. The second strategy is thus to design a structure with hexagons, which is always stable, but offers less design freedom. The last design strategy uses post-rationalization to cover doubly-curved shapes with planar facets.

\subsection{Origami}

Connections between plated structures and origami were made by Buri and Weinand [2], as origami naturally generate creases, which can be interesting for the structural behaviour of plated structures. Origami is also an active field of study and can be of interest for builders, as rigid origami are covered with planar facets and simplify manufacturing.

The topic of folded structures for architecture has been studied extensively, a review of this topic is proposed by Lebée [3], and some examples apply to folded plate structures. For example, Tachi generalised the form-finding of the Miura-Ori folding pattern with planar quadrilateral and proposed a design for a barrel vault [4]. Recent examples of folded plate structures include planar quadrilateral meshes generated with a principle using two curves $[2,5]$. Notice that some rigid origami are rigorously described by the means of discrete differential geometry. For example, Schief et al. [6] studied the egg-box pattern and its foldability, naming the resulting meshes 'discrete Voss surface'.

\subsection{Hexagonal pattern}

It is a well-known fact that trivalent plates generally form a stable plated system. For example, origami requires a folding pattern which is two colourable, and folding of a trivalent system requires bending or twisting at least one plate [3]. Several built examples use this stability property, like the TRADA Pavilion [7] and a pavilion built for the Landesgartenschau [8]. While the latter building is a success, Knippers and Li reveal potential pitfalls when covering a shape with planar hexagons [8]. Meshing with planar hexagons implies indeed following lines of curvatures, and the hexagons tend to align with Dupin's indicatrix. In negative curvature area, the panels take a bow tie shape. This implies poor nesting and large wastes generated. Acute angles resulting from the bow-tie shape can also be problematic in terms of construction.

Beside, hexagonal meshing does not guarantee stability in any situation, especially in areas with zero gaussian curvature [8]. Unlike quadrilateral patterns, hexagonal patterns cannot be creased and therefore do not benefit from a high equivalent bending stiffness.

\subsection{Post-rationalization}

During the last decade, significant progress has been made in the field of architectural geometry for the rationalization of construction. Optimization methods can be used to approximate a shape by a mesh covered with planar panels. For the design of 
PQ meshes, the input mesh has to follow conjugate curve networks, so that the designer does not control the topology of the pattern. If the fabrication process allows some non-planarity of the plates, the designer can use other curve networks on a target surface and perform optimization towards planarity of the facets. In [9], Robeller and Weinand use the ShapeOp optimization framework to reach facet planarity. Because the initialization does not follow a conjugate curve network, they introduce eccentricities to cope with the non-planarity of the mesh resulting from optimization.

There is little feedback on the use of postrationalization for the design of folded plate structures, due to the novelty of the approach. We point out two possible limitations. First, the introduction of eccentricities into plated shells is expected to decrease their bearing capacity, as they are geometrical imperfections. Second, optimization is suited for smooth target surfaces, so that corrugation cannot be introduced easily with this design methodology. Robeller and Weinand [9] deal with this issue by constructing a double-layer plated structure.

\subsection{Structural performance}

Creased structures are often considered to be highly efficient. In a study on cylindrical corrugated shell, Buri and Weinand [2] claim that 'the higher the amplitude, the stronger the resistance of the folded plate structure'. This statement is well documented in the case of flat geometries or arches [10], which were studied by Buri and Weinand. Indeed, creases introduce an equivalent moment of inertia larger than that of the single elements. In structures subject to biaxial stresses, the influence of creases is however not so obvious as it decreases the axial stiffness in the transverse direction.

A study comparing the performance of different patterns for folded plate structures applied to a cylindrical shell was proposed in [10]. It demonstrates that the Miura Ori pattern is subject to much higher deflection than patterns based on trapezoids or the Yoshimura pattern. The choice of an appropriate corrugation strategy is therefore crucial, but the lack of a unifying framework makes the comparison of solutions difficult. Other case studies on wooden plated structures have been made and successful demonstrators were built in recent years $[2,10,12]$, but the difficulty to parameterise a design space of feasible solutions with planar quadrilateral facets has limited the generality of these studies.

A multi-objective exploration of a design space of folded plate structures was performed with genetic algorithms in $[13,14]$. The proposed design space was however restricted to spherical shapes or to triangular meshes, which are less stable than hexagonal or quadrilateral patterns.

\subsection{Problem statement}

The objective of the present paper is to introduce a general framework for the modelling of plated structures covered with planar panels. The proposed method is not restricted to origami, and unifies some of the previous design strategies described in [10]. The shape-generation strategy can also be extended to non-standard patterns, like the Kagome [15], although this paper focuses on quad patterns.

The generality of the method allows parameterising structural optimisation problems efficiently. Therefore several case studies are conducted to compare different creasing strategies for quadrilateral meshes derived from our framework.

\section{METHODOLOGY}

\subsection{Shape generation with marionette meshes}

\section{Description of the method}

This article proposes to unify previous methodologies for the generation of corrugated structures. The main technological constraint is to construct shapes with planar facets, which is nontrivial for quadrangular panels. We use here a framework for shape generation introduced and called marionette meshes by the authors [16]. This method is highly intuitive and computationally efficient, and based on descriptive geometry. It is shown in [16] that the user can prescribe a projection (say in the horizontal plane) and several elevation curves of a mesh to construct a unique mesh with planar faces. The lifted mesh is simply found by solving a linear system. An example of input data yielding exactly one mesh with planar facets is displayed in Figure 2.

The method also allows reformulating previous strategies for shape-generation of plated structures. Corrugation can indeed be applied to smooth 
meshes by introducing perturbations in the plane view or in the elevation curves. In the followings, we introduce two corrugation strategies based on this principle.

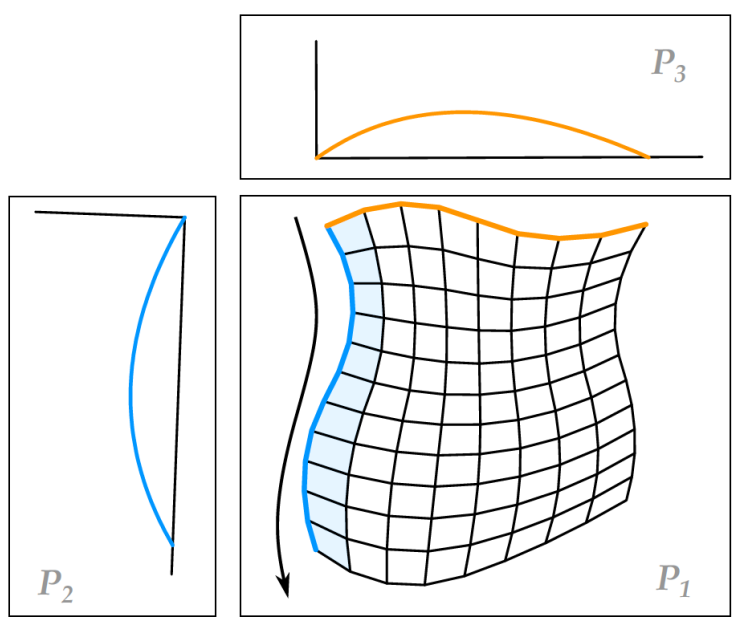

Figure 2: One planar projection and two elevation curves determine a unique quad mesh covered with planar facets (image: [16])

\section{Row-Translation strategy}

In [10], the Miura Ori strategy corresponds to the approximation of a cylinder by a Miura Ori pattern. The solution is made of rhombi. In Figure 3, a simple way to generate a network of parallelograms from a square grid is proposed. Starting from a reference mesh, the method consists in assigning a displacement to each row of the planar view and then to apply the marionette technique to the transformed planar view.
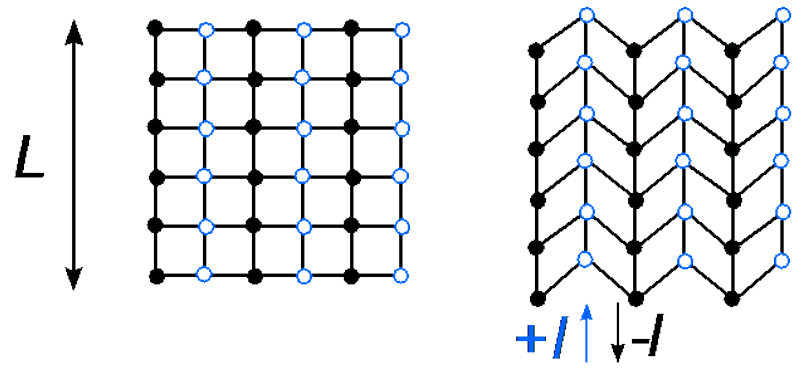

Figure 3: Strategy for the generation of 'Miura Ori' (herringbone) pattern

Two parameters are identified to control the corrugation:

- The in-plane corrugation length $l$, corresponding to the amplitude of the displacement for each row, represented in Figure 3;
- The corrugation of the elevation $\delta_{H}$, corresponding to the amplitude of the corrugation for one elevation curve.

In the general case, this strategy does not yield a foldable pattern. We thus denote it as rowtranslation (RT) strategy instead of Miura-Ori strategy, which makes explicitly reference to origami and foldable structures.

\section{Alternate-translation strategy}

In [10], a pattern of trapezoids is made on a cylindrical shape. The pattern is generated by local modification of a triangular pattern, but it could be generated directly with a method illustrated in Figure 4: the points of the quadrilateral pattern are alternatively translated in two opposite directions.

Unlike the Miura-Ori pattern, the elevation curves remain smooth, which leaves two parameters:

- $\quad$ The in-plane crease amplitude $l$;

- The crease direction, which corresponds to the angle $\theta$ made by the translation with the edges of the initial mesh.

By reference to the other proposed strategy, we call this strategy the alternate-translation (AT) strategy.
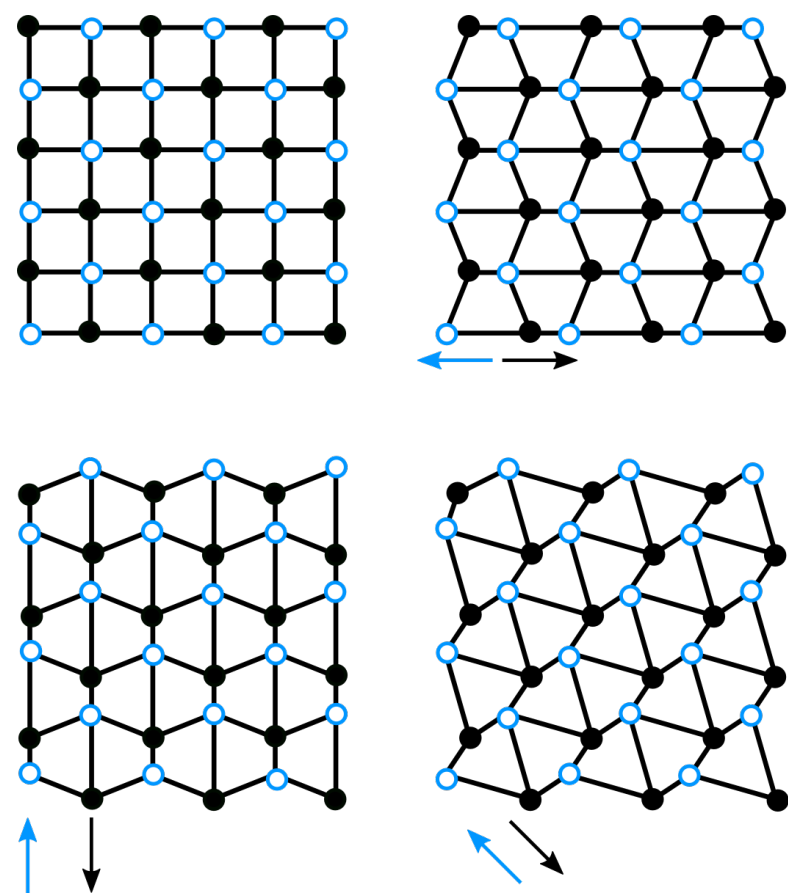

Figure 4: Strategy for the generation of 'trapezoid' pattern 
Controlling complex geometry with NURBS modelling

The geometrical rules underlying creasing strategies make the parameterisation of the design space very light.

The choice of the amplitude can be made with respect to mechanical considerations. Current literature on folded-plate structures makes references on an equivalent structural depth to withstand bending. Corrugation is also expected to decrease the axial stiffness in the transverse direction, but the influence of the corrugation amplitude is less discussed.

\subsection{Definition of the geometry}

This article implements the marionette method to generate a design space of folded plate structures covered with planar facets. The two studied geometries are represented in Figure 5: they are both derived from a network with two singularities of type 'lemon'. One is a dome without opening, and the other one is obtained by the removal of one sector of the mesh. The two singularities in the closed dome allow for alignment of the mesh on the boundaries, which is meaningful when using creasing strategies: they also illustrate the formal potential of the marionette technique, which allows for the modelling of complex topologies, as shown also in Figure 1.
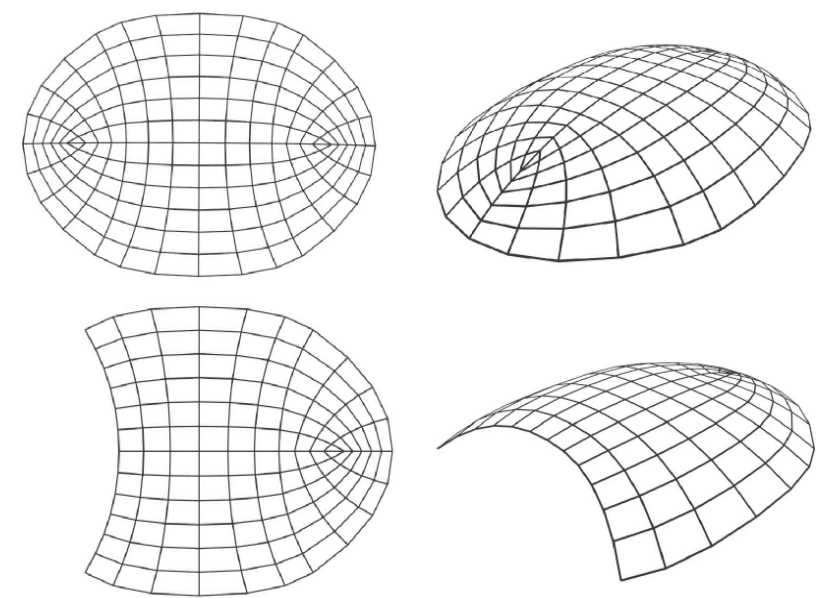

Figure 5: The two configuration studied: a closed dome and a dome with a free edge

The two models are expected to behave differently, as free-edges are known to be a critical issue in thin shell design. The plane view is parameterised with
NURBS patches, but is not a parameter of our study. The in-plane creasing strategies are specified with respect to the local coordinate axis of the patches $\boldsymbol{t}_{\boldsymbol{u}}$ and $\boldsymbol{t}_{\boldsymbol{v}}$. For a general NURBS patch, the translation vector is given by:

$$
\boldsymbol{T}(\boldsymbol{u}, \boldsymbol{v})=l\left(\cos \theta \boldsymbol{t}_{\boldsymbol{u}}+\sin \theta \boldsymbol{t}_{\boldsymbol{v}}\right)
$$

The two corrugation strategies introduced in this article are compared. Figure 6 represents two plane views of the corrugated domes generated with the AT and RT strategies. Coloured lines correspond to lines with an elevation prescribed with the marionette technique. The initial configuration has an axis of symmetry represented as a thick black line in Figure 6: we choose here to preserve the symmetry. Two singularities of type "lemon" have been introduced in order to align the mesh with the supports and avoid boundary effects in our structural investigations. The two creasing strategies proposed in this article require adjustments due to the introduction of these singularities. For example, the principle of the AT strategy works if one is able to separate two families of points. The meshes considered in this paper are 2-colourable, but applying both strategies would yield non-symmetrical shells and break the symmetry of the initial geometry. The mirroring operation performed here is thus also motivated by topological considerations.

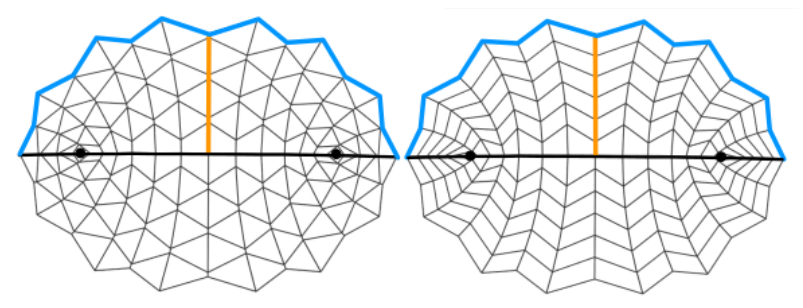

Figure 6: The two corrugation strategies applied to the planar view: AT (left) and RT (right), black dots indicate the two singularities

\subsection{Loads, materials, boundary conditions}

The material chosen has a Young's modulus of $10.5 \mathrm{GPa}$. It is no more than a scaling factor when linearized buckling or linear analysis are considered. The boundary conditions are set as hinges along the edges and translations are fully restrained.

In shell design, the governing load case is often a non-symmetrical one (like snow or wind loads for example), as it activates bending moments in the 
structure. Therefore, we consider a vertical pressure applied on one half of the structure as depicted in Figure 7.

Two mechanical connections between plates are considered: hinges or moment connections. Details on the implementation with the finite element method are given in Section 3.5.

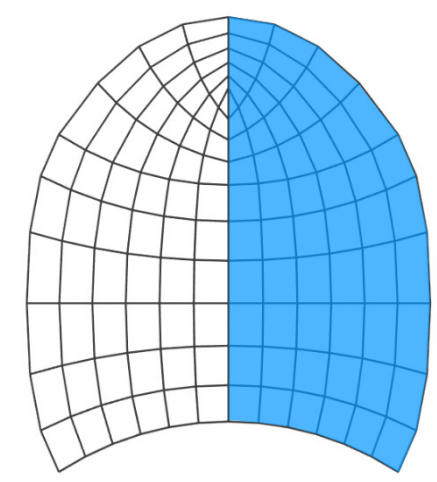

Figure 7: Load distribution: the vertical load is applied to the blue area only

\subsection{Non-dimensional analysis}

The structural behaviour of folded plate structures is assessed with two performance metrics: the maximal displacement $\delta$ under a given load $p$ and the linearized buckling factor $p_{c r}$. These indicators are useful in conceptual design stages. The detailed design of plated structures requires full non-linear calculations with geometrical imperfections and possibly elasto-plastic design, in the manner of gridshell structures $[19,20,21]$.

Like in many classical works, we present the results with non-dimensional parameters. It has the advantage of reducing the number of parameters thanks to the application of Buckingham $\Pi-$ Theorem. The geometries described in this paper can be characterised by a span $L$, a height $h$ and a thickness $t$. We add the amplitude $l$ of the in-plane corrugation vector $T(\boldsymbol{u}, \boldsymbol{v})$ defined by equation (1). The mesh has $n \times m$ subdivisions. The material is characterised by its Young modulus $E$ and Poisson's ratio $v$ (more parameters can arise when considering orthotropic materials).

There are thus seven physical variables, and two units. We can construct five dimensionless parameters that uniquely define the problem.
The first one is the rise-over-span ratio, which is typically between $15 \%$ and $40 \%$ for gridshells [14]. We chose two values for this ratio: $15 \%$ and $35 \%$.

$$
\Pi_{1}=\frac{h}{L}
$$

The second is the corrugation ratio, which indicates the level of corrugation in the surface. It can take values between 0 and 0.5 .

$$
\Pi_{2}=\frac{n \cdot l}{L}
$$

As an example, Figure 6 shows four ellipsoidal shapes with varying corrugation ratios, all other parameters being equal. The overall shape is unchanged, but local crumbling is added, creating large ribs. The geometries displayed in Figure 8 are studied in Section 4.1.

The third ratio is the slenderness, typically below $1 \%$ for applications in high-performance shells.

$$
\Pi_{3}=\frac{t}{L}
$$

Higher values of $\Pi_{3}$ correspond to higher bending stiffness, relatively to the axial stiffness. Indeed, writing $\mathcal{D}$ and $\mathcal{A}$ the bending and axial stiffness of the plate, the quantity introduced in equation 4 can be described by:

$$
\Pi_{3} \propto \sqrt{\frac{\mathcal{D}}{\mathcal{A L} L^{2}}}
$$

The next two ratios correspond to non-dimensional buckling load and displacement. They are the performance metric studied in this paper.

$$
\begin{aligned}
& \Pi_{4}=\frac{p_{c r} L^{3}}{E t^{3}} \\
& \Pi_{5}=\frac{\delta E t^{2}}{p L^{3}}
\end{aligned}
$$

The results are not normalised with respect to the mass, like usually done in studies on structural efficiency $[11,15]$. The mass of the corrugated structures varies indeed by less than $1 \%$, so that $\Pi_{4}$ and $\Pi_{5}$ can be considered as reliable indicators of structural efficiency. In the following, we set the span $L$ and the Young's modulus $E$ as constants. The other parameters can be varied. 

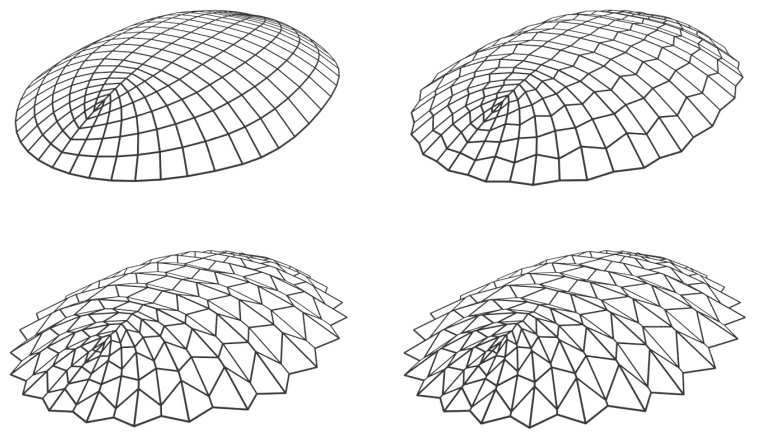

Figure 8: Domes generated with AT strategy, with different corrugation ratios (from left to right and top to bottom: $\left.\Pi_{2}=0,0.1,0.3,0.4\right)$

\subsection{Finite element modelling}

\section{General considerations}

The structural behaviour of the folded plate structures generated by our method is evaluated with the finite element method. We use the structural software Karamba [17], which implements shell elements [18]. The attachment between the panels is described in Figure 9. Each plate is slightly shrunk and subdivided, and springs are introduced between the duplicated nodes. The local $z$ axis of each spring is aligned with the edge boundary (orange arrow in Figure 9). In that way the force $F_{z}$ corresponds to in-plane shear, $F_{y}$ corresponds to out-of-plane shear. The links restrict translations, and allow rotations along $x$ and $y$ axis. Rotations are restricted along the $z$ axis.

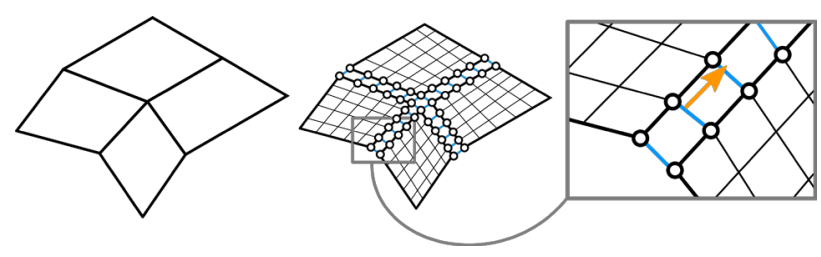

Figure 9: Model of structural attachment: initial geometry (left), shrunk plates with subdivision (middle) and links between the duplicated nodes (right).

\section{Convergence study on link stiffness}

The linkage between the plates is made with spring elements. The spring axial and shear stiffness are not a parameter of our study, but could be taken into account depending on the type of connection used. Our study considers springs that act as rigid links for the translational degrees of freedom. Figure 10 shows the influence of the axial and shear stiffness on the overall displacement of a plated structure under a uniform live load. It is noticed than when increasing the axial and shear stiffness, the maximal displacement converges to a certain value. The springs act then as a rigid link. The spring stiffness was thus set accordingly to this convergence graph at $10^{7} \mathrm{~N} / \mathrm{m}$.

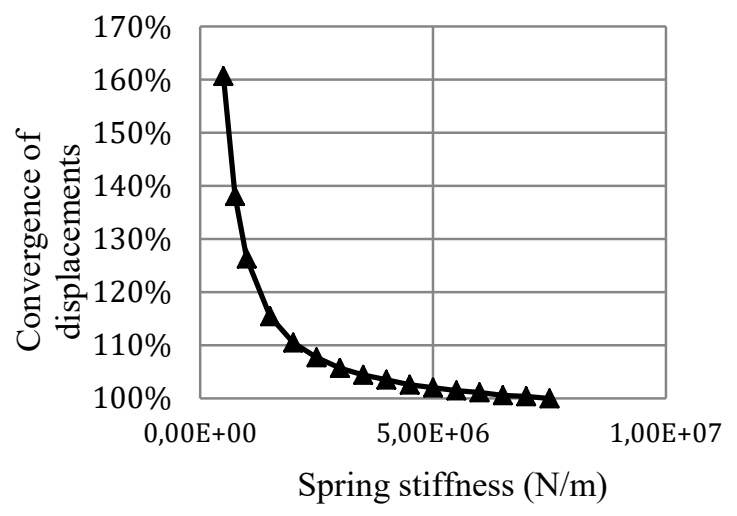

Figure 10: Influence of spring stiffness on displacements

\section{Convergence study on mesh refinement}

The convergence of the finite element model with mesh refinement is studied with a plated shell. Figure 11 shows the convergence of elastic energy and maximal displacement with respect to the number of subdivisions along each edge $N$. The total number of elements varies with the square of $N$. Displacement and energy are compared to a reference value, set for 15 subdivisions of each edge, which corresponds to 79,000 elements. The graph shows that convergence of the model is quickly reached. The convergence of elastic energy and displacements is above $95 \%$ for 6 subdivisions per edge. We choose this refinement value in the following, as it is a good compromise between convergence and computational efficiency.

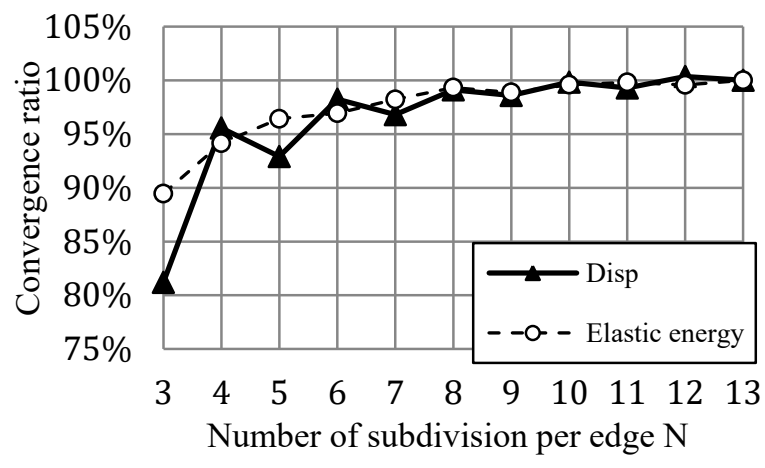

Figure 11: Influence of mesh refinement on convergence 


\section{STABILITY OF FOLDED PLATE STRUCTURES}

This section presents the results of the parametric study aiming at evaluating the structural performances of corrugated shell structures. Only the results for $h / L=0.15$ are shown in this Section, other results for $h / L=0.35$ are presented in the Appendix. A discussion and practical design guidelines are proposed in Section 5.

\subsection{Case-study: closed dome}

\section{Results of the AT strategy}

The results of the linearized buckling calculation for a closed ellipsoidal dome are displayed in Figure 12. The parameter studied is the corrugation factor and the performance metric represented is the linear buckling load. Low values of $\Pi_{2}$ indicate smooth geometry and values of $\Pi_{2}$ close to 0.5 indicate highly creased structures (see Figure 8).

Plates with rigid connections have a substantially higher bearing capacity than hinged plates for geometries without corrugations. Their bearing capacity is however less affected by corrugation: the critical buckling load increases by approximately $30 \%$ for rigidly connected shells, and up to a factor 10 for hinged plates.

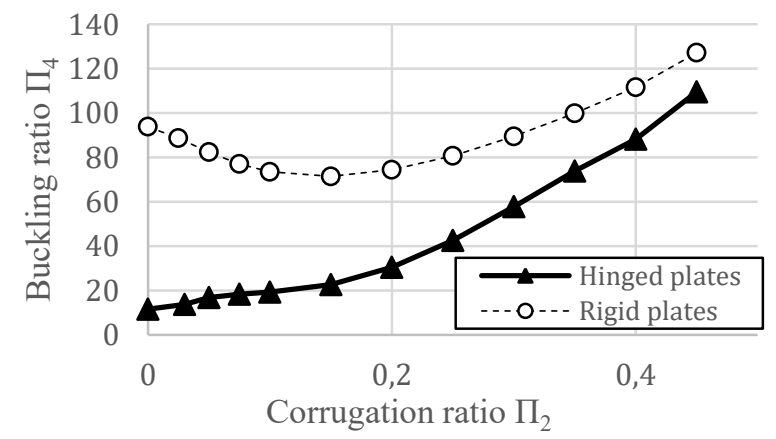

Figure 12: Buckling load vs. corrugation factor $(t / L=1 \%$, $h / L=0.15$, AT strategy)

Low levels of corrugation appear to decrease the bearing capacity of structures with rigid connections, with a minimum reached for $\Pi_{2}=$ 0.15 . For higher values of $\Pi_{2}$, the corrugation increases the structural performance. The effect of corrugation could thus be interpreted as a geometrical imperfection applied to slender structures, like gridshells $[15,19,20]$.
Figure 13 shows that the ratio of the critical buckling loads $\frac{p_{c r, \text { rigid }}}{p_{\text {cr,hinged }}}$ decreases with the corrugation amplitude. This tendency shows that the corrugation creates a geometrical interlocking of the plates, which has a rigidifying action.

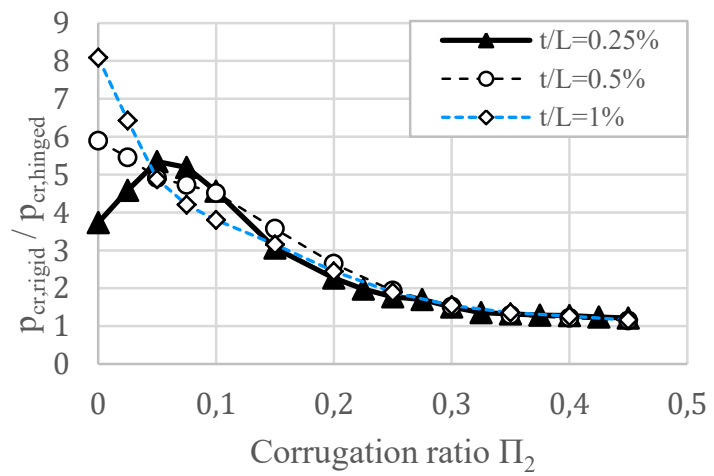

Figure 13: Ratio of the linearized buckling loads for the rigid and hinged plates configurations $(h / L=0.15)$

The results on maximal displacements indicate that structures with small corrugations are less rigid than the reference geometry. Figure 14 shows the nondimensional displacement in function of the corrugation factor. Past a certain corrugation factor (with $\Pi_{2}$ from 0.1 to 0.2 ), the displacements decrease with corrugation. The stiffest configuration remains the initial one in our study: if only maximal displacement is considered, the interest of crease is therefore inexistent for the considered case study. Recall that corrugation of creased structures increases the bending stiffness in one direction, but decreases the axial stiffness transverse to that direction. We can interpret the curve as follows: first, the axial stiffness diminishes in the transverse direction with small corrugations, but is not compensated by the increase of the bending stiffness. For sufficiently high corrugations, the stress distribution becomes mainly uniaxial and the bending stiffness introduced by the creases governs the structural response.

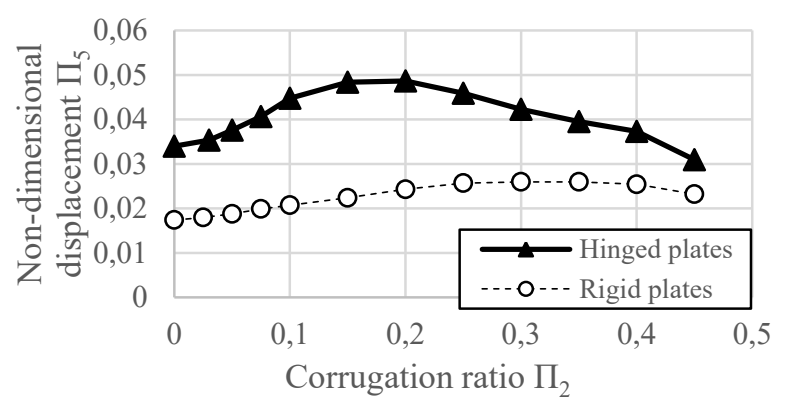

Figure 14: Non-dimensional displacement vs. corrugation factor $(h / L=0.15, t / L=1 \%$, AT strategy) 
It should be noted however that high corrugation (superior to 0.2) diminishes differences between hinged and rigid connections, as illustrated in Figure 15.

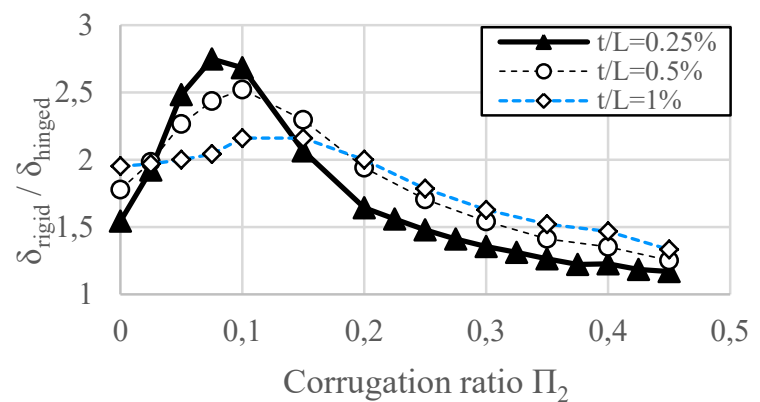

Figure 15: Ratio of the maximal displacements for hinged and rigid plates (AT strategy, $h / L=0.15$ )

Results with another rise-over-span ratio do not fundamentally differ from the presented results. It can just be noted that the ratio $\frac{p_{c r, \text { rigid }}}{p_{\text {cr, hinged }}}$ decreases with the rise-over span ratio for low corrugation levels. Indeed, shallow shells have a lower geometrical stiffness than deep shells, and rely thus more on their bending stiffness.

\section{Results of the RT Strategy}

Plated structures meshed with the RT strategy behave differently from the ones meshed with the AT strategy. Hinged plates are indeed substantially less efficient than rigid plates for any level of corrugation, as shown in Figure 16 and in the Appendix 1. In some cases, the corrugation even decreases systematically the buckling capacity of folded plate structures.

The rigid plates reach the same buckling load as with the AT strategy for high corrugations. Plates with hinged connections however have a lower buckling load. This would advocate for the choice of the AT strategy, but the RT strategy yields displacements lower by $50 \%$. Consider for example Figure 17: the lowest non-dimensional displacement obtained for the hinged plates is 0.021 (for $\Pi_{2}=0.45$ ), which is lower than the minimum reached for the AT strategy shown in Figure 14 (minimum non-dimensional displacement of 0.031). The choice of the most appropriate corrugation strategy is thus a matter of multi-criteria optimization. The detailed analysis of the governing design criterion is needed to determine the most interesting corrugation strategy.

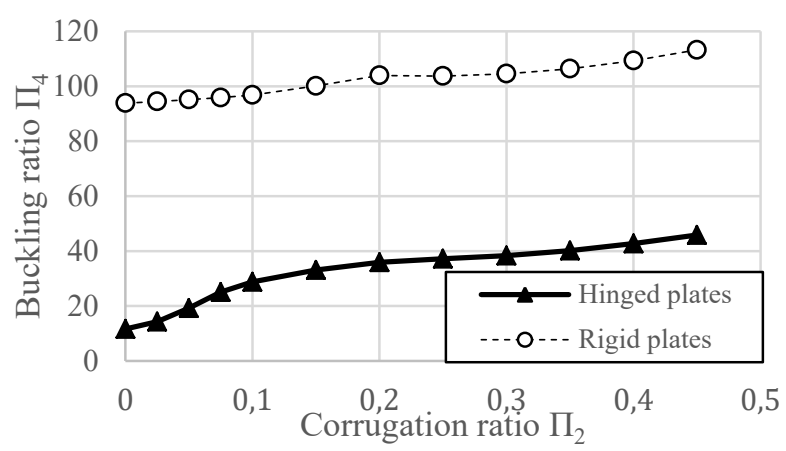

Figure 16: Buckling load vs. corrugation factor $(t / L=1 \%$, $h / L=0.15$, RT strategy

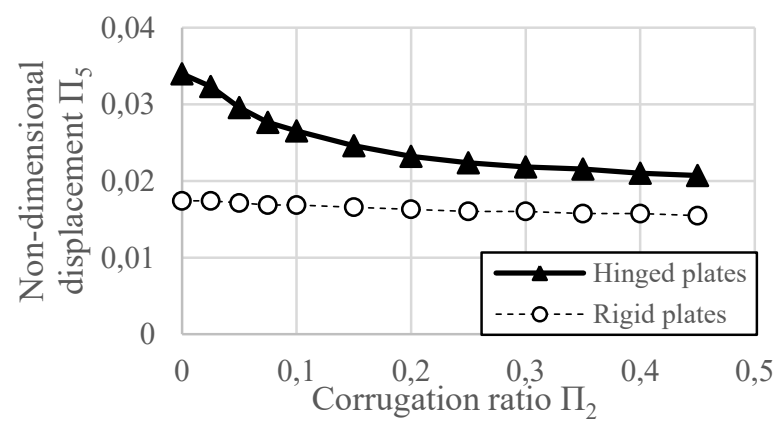

Figure 17: Non-dimensional displacement vs. corrugation factor $(t / L=1 \%, h / L=0.15$, RT strategy

Figure 18 represents the ratio between maximal displacements of folded plate structures with hinges or moment connections. This ratio increases for low levels of corrugations, and decreases after a peak reached approximately for $\Pi_{2}=0.2$.

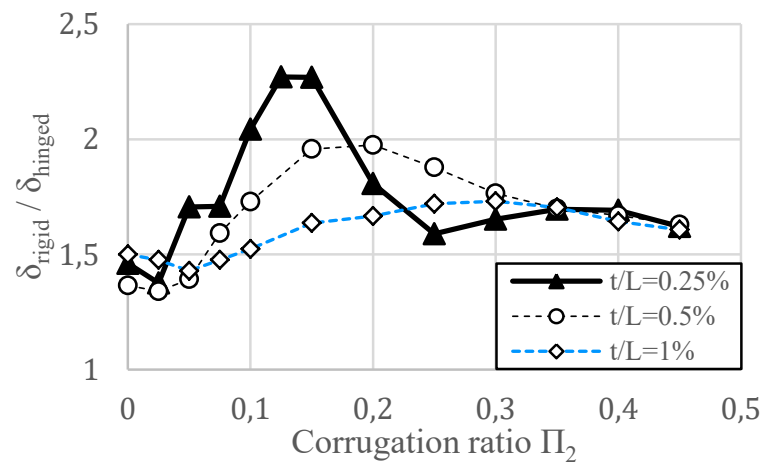

Figure 18: Ratio of the maximal displacements for hinged and rigid plates (RT strategy, $h / L=0.15$ )

\subsection{Case-study: dome with free-edge}

Results of the AT strategy

Consider now the same elliptical dome where an opening has been created, such as shown in Figure 
5. The resulting free-edge changes the structural response of the shell under non-symmetrical loads.

Unlike the closed dome, the corrugation improves significantly the bearing capacity of both hinged and rigid structures, as shown in Figure 19 and 20. Compared to the reference geometry, the critical buckling load is multiplied by up to 4 .

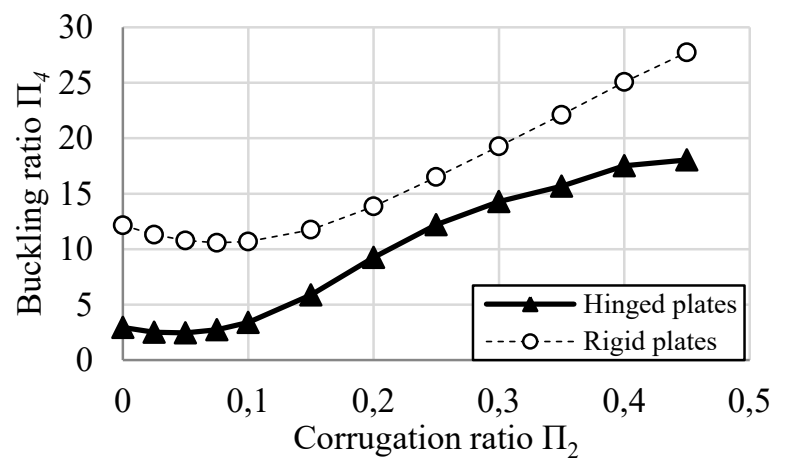

Figure 19: Buckling load vs. corrugation factor $(t / L=1 \%$, $h / L=0.15$, AT strategy)

Figure 20 shows the ratio $\frac{p_{c r, \text { rigid }}}{p_{c r, \text { hinged }}}$ with respect to the corrugation factor. The ratio converges quickly to a value around 1.3. The curve features a plateau for corrugation factors superior to 0.2 , which is not observed for the RT strategy as shown in Figure 22.

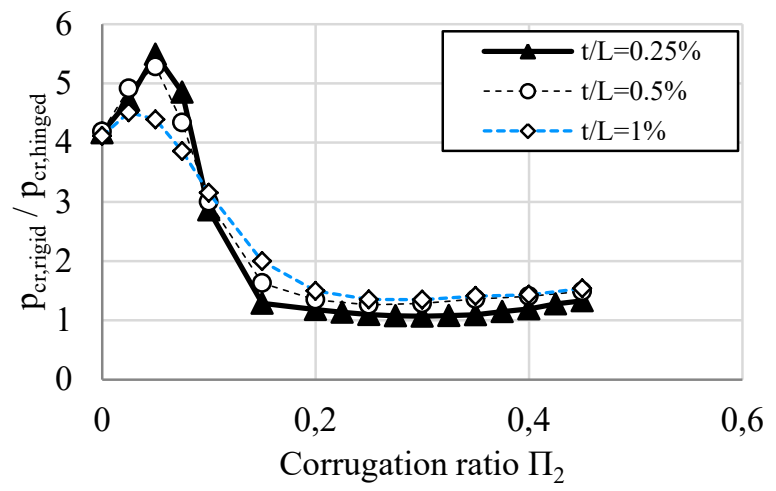

Figure 20: Ratio of the linearized buckling loads for the rigid and hinged plates configurations $(h / L=0.15)$

Corrugation positively affects the bearing capacity of plated shell structures, but also their stiffness, as illustrated in Figure 21, which represents maximal non-dimensional displacements for all the tested configurations. A global minimum is reached for moderate value of $\Pi_{2}$. The stiffest structures are up to seven times stiffer than the structures without corrugation. We notice that the curves are non- convex, with several local minima (at $\Pi_{2}=$ $0,0.225,0.4$ for hinged plates).

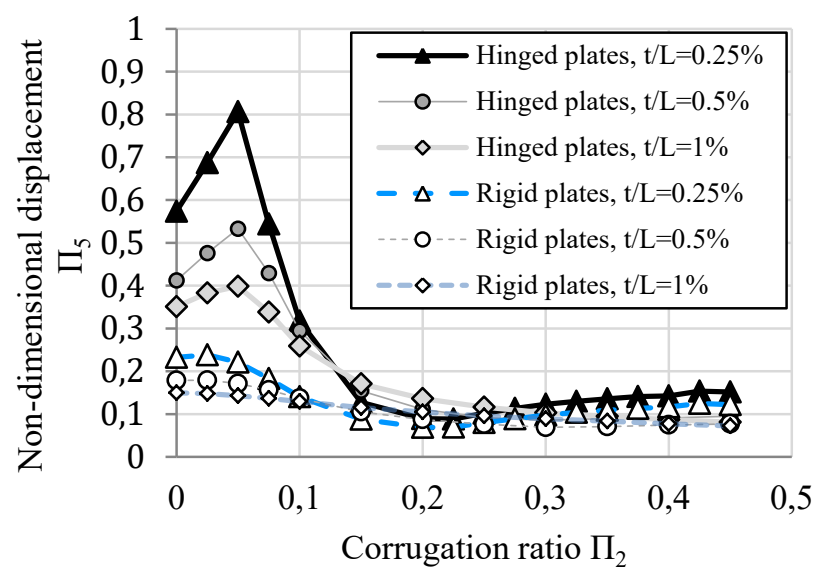

Figure 21: Non-dimensional displacements vs. corrugation ratio $(h / L=0.15)$

\section{Results of the RT strategy}

The RT and AT strategy yields similar results for the linear buckling analysis. The bearing capacity of plated structures is improved by corrugation, as shown in Figure 22.

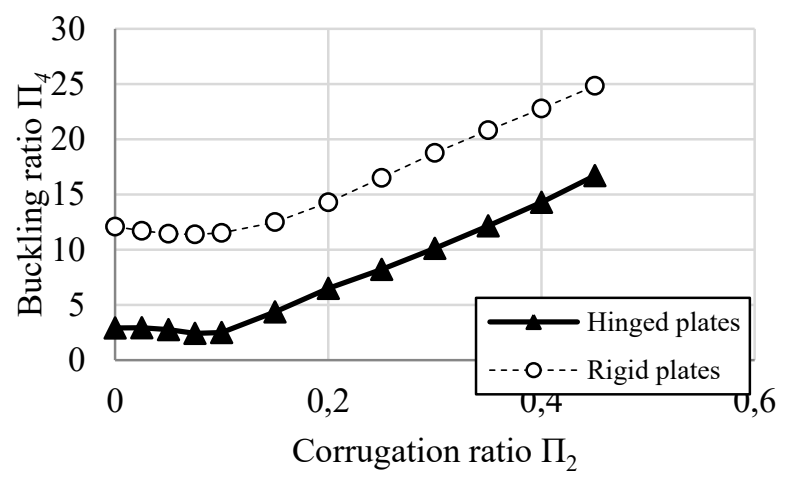

Figure 22: Buckling load vs. corrugation factor $(t / L=1 \%$, $h / L=0.15, R T$ strategy)

Figure 23 displays the ratio $\frac{\mathrm{p}_{\mathrm{cr}, \text { rigid }}}{\mathrm{p}_{\mathrm{cr} \text {,hinged }}}$ in function of the corrugation ratio. For low corrugation levels, plates with rigid attachment have a much higher linear buckling load, but the ratio $\frac{p_{\text {cr,rigid }}}{p_{\text {cr,hinged }}}$ decreases down to 1.4 for high corrugations. The difference between hinged plates and rigid plates is therefore still important, although the convergence shows that the corrugation decreases the sensitivity to the stiffness of the mechanical attachment between plates. 


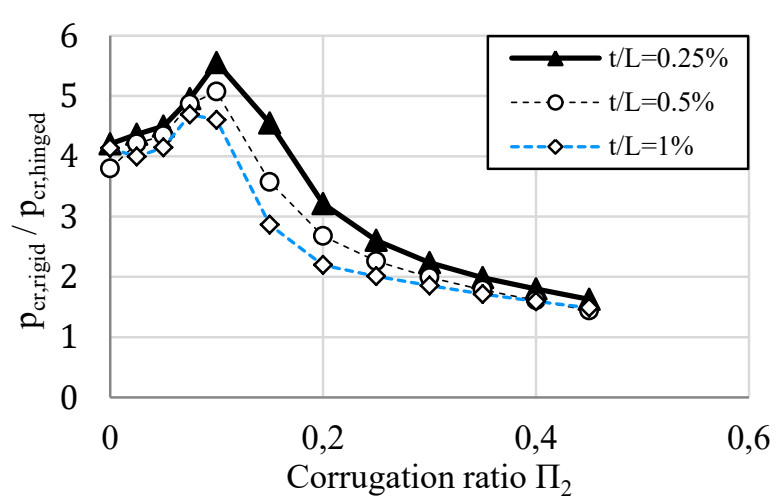

Figure 23: Ratio of the linearized buckling loads for the rigid and hinged plates configurations $(h / L=0.15)$

The main difference between the AT and RT strategy concerns the influence of the corrugation on the maximal displacements. Figure 24 shows that the ratio $\Pi_{5}$ decreases less for the RT strategy than for the AT strategy: there is no local minimum for the curves shown in Figure 24. The corrugated structures can be up to 2 times stiffer than the noncorrugated ones, which still validates the use of corrugation for shells with free edges.

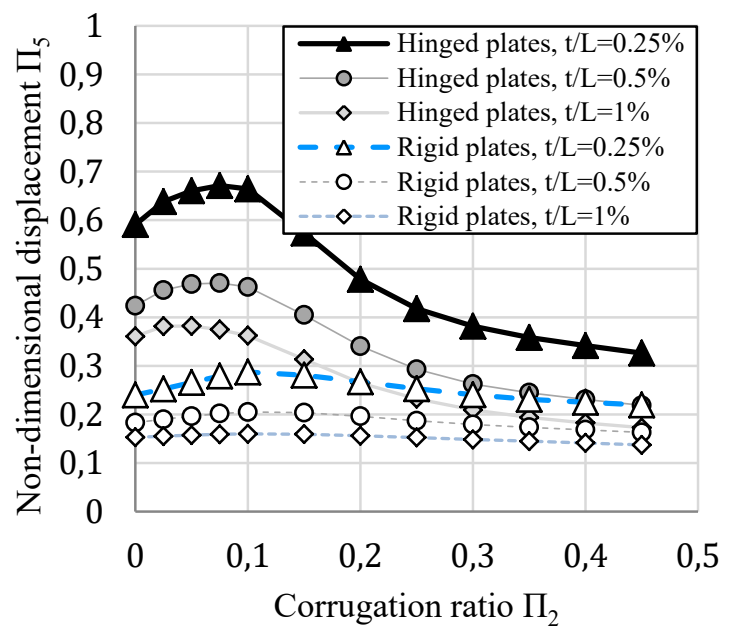

Figure 24: Non-dimensional displacements vs. corrugation ratio $(h / L=0.15)$

\subsection{Influence of rise-over-span ratio}

The previous section presented results for shallow shells $\left(\Pi_{1}=0.15\right)$; another rise-over span ratio was studied $\left(\Pi_{1}=0.35\right)$. The same overall tendency can be observed: corrugation improves the buckling capacity and stiffness of structures with a free-edge. The qualitative differences between the two corrugation strategies discussed hereinabove are also observed for other rise-over-span ratios.
Like already noticed in previous studies [11,20,21], increasing the rise-over span ratio improves both stiffness and linear buckling load. This illustrates that plated shell structures act well under membrane action.

\section{DISCUSSION}

\subsection{Comparison the two proposed strategies}

The present parametric study considered two different performance metrics: displacement and linear buckling load. Structural engineers aim at minimizing the displacements and at maximizing the linear buckling load. It is usually impossible to find a solution that is optimal for all performance metrics, so that we need to use notions specific to multi-criteria optimisation to compare the quality of the two design spaces generated with the different creasing strategies.

Therefore, in the following we will say that a design is dominated if there is another design with better performance for every performance criterion (i.e. lower displacement and higher buckling load). We use the Pareto frontier in order to compare the two corrugation strategies. A Pareto frontier contains all the non-dominated solutions. They are optimal in the sense that it is not possible to improve the performance on one metric without decreasing it for the other (it is not possible to find designs that would have both a higher buckling capacity and a higher stiffness). Figures 25 and 26 show some of the data generated in our study. In those Figures, the designer would tend to seek designs towards the top (high buckling capacity) and left (low displacement). No data can be found in the top left quadrant of Pareto optima.

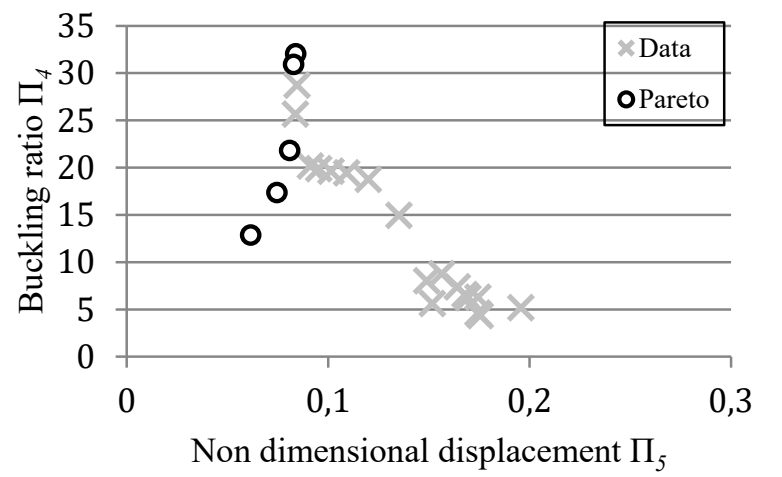

Figure 25: Pareto frontier for the open dome, $t / L=1 \%$ with hinged plates 


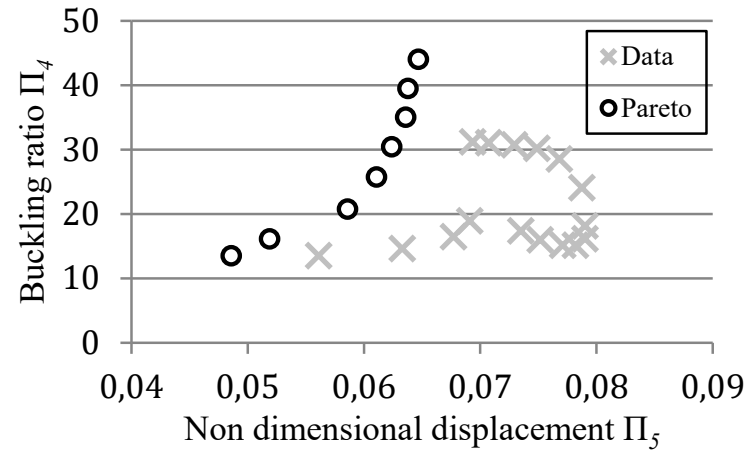

Figure 26: Pareto frontier for the open dome, $t / L=1 \%$, with rigid plates

In Figures 25 and 26, Pareto optimal designs are generated with the AT strategy. This is a general observation for the shell with a free edge. For moderate corrugations, the AT strategy also yields significantly stiffer structures (by a factor 3 approximately). It was already observed in [11] on one specific arch design for a symmetrical load.

As already discussed in Section 4.2, the closed dome configurations have Pareto optima in both the AT and RT design space. The AT strategy increases significantly the linear buckling load while the RT strategy improves the structural stiffness. The parametric study is thus less concluding for the closed domes, as the optimal strategy depends on the weighting of the two factors.

\subsection{Design guidelines}

Creased structures offer a design space that has not yet revealed its full potential. The numerical exploration of the space of feasible design is eased with our simple strategies that require few parameters. The computational cost for a systematic study on the influence of corrugation remains however high, as mesh refinements are inevitable and lead to large models. The case studies performed in this paper allow us to provide some guidelines for future design explorations with plated shell structures.

First, the numerical study demonstrates that plated structures are highly sensitive to imperfections. A small change in the initial geometry can greatly influence the buckling capacity of plated shells, especially for non-corrugated shapes. As corrugation increases, the structure transitions from shell behaviour to arch behaviour. Indeed, curved corrugated structures loose stiffness in the transverse direction, as illustrated in [22]. The structural performance of plated shells with moderate values of corrugation is thus poor, since it does not benefit of two-way stress distribution or large equivalent bending stiffness.

Second, it appears that creased plated shells are efficient for structures with a uniaxial stress state. For example, barrel vaults are subject mainly to uniaxial stresses, and the corrugation improves significantly the structural behaviour. On the contrary, creasing does not seem to necessarily be a meaningful strategy when the smooth structures rely on biaxial stress state. In those cases, the geometry forces the loads to follow the ridges and valleys of the corrugation, turning a biaxial stress state into a uniaxial one. In our case study on domes, corrugated structures are less rigid than noncorrugated ones. Therefore, creasing strategies should be used in priority for structures with a privileged spanning direction. Examples of such structures include barrel vaults, toric shapes like stadia, shells with free edges or shells on punctual supports.

Third, it is noticed that the rotational stiffness between plates influences the overall structural behaviour, especially for low levels of corrugation. Configurations with fully connected plates are more rigid, but are more difficult to fabricate. Our strategy introduces configurations that are not foldable, and guarantees the stability of structures even with hinges between plates.

Finally, we have seen that different local optima appear when varying the corrugation amplitude (consider the remark made for Figure 21), so that the objective functions associated to the performance metrics used in this paper are not convex. Furthermore, the data generated in this study considered mainly the influence of corrugation on the structural performances of plated shell structures. This advocates for the use of global optimisation algorithms for the structural optimisation of plated shell structures and validates the approach used in $[13,14]$.

\subsection{Exploring patterns}

This work focused on quadrilateral meshes, as it is commonly used for folded plate structures. The marionette method can however be generalized to other patterns, as illustrated in [16]. 
Patterns mixing valence four and three, like the Cairo tiling could be of interest in future work. The valence three would provide stability for hinged plates whereas the valence four makes the pattern more flexible than hexagonal pattern.

A post rationalisation technique for the modelling of semi-regular meshes with planar facets has been proposed in [23]. The method requires however good initial input, for example when the mesh layout follows the lines of curvature of the surface or conjugate curve network. The marionette technique and other bottom-up design strategies [24] should be investigated to provide such parameterisations.

\section{CONCLUSION}

This paper introduced a general framework for the modelling of plated shell structures. Two corrugation strategies were introduced to generate creased structures with planar quadrilateral facets. The design approach proposed in this paper is based on the marionette technique which guarantees exact facet planarity and simplifies their fabrication. Depending on the choice of assembly technology, other fabrication constraints, like edge length uniformity or nesting could be considered.

A parametric study investigated the influence of crease on the structural behaviour of plated shell structures for simple performance metrics. The study illustrates the influence of the crease amplitude on the overall structural behaviour of plated shell structures. Creasing strategies are particularly meaningful if the structures feature free edges, or if the plates are hinged.

The present study considered both hinged and rigid connections. Different technologies for the assembly of folded plate structures exist: their choice is likely to influence greatly the structural response of folded plate structures. Physical tests remain necessary to evaluate their bearing capacity and stiffness. We see a potential in exploring hinged connections, which could easily be assembled. An example of application could be the fabrication of reusable formwork for concrete shells or temporary structures.

\section{ACKNOWLEDGMENTS}

This work was made during Mr. Mesnil's doctorate within the framework of an industrial agreement for training through research (CIFRE number 2013/1266) jointly financed by the company Bouygues Construction SA and the National Association for Research and Technology (ANRT) of France.

\section{REFERENCES}

[1] Arpad K., Folded plate structures and components therefor, US Patent, 3557501; 1971.

[2] Buri H., Weinand Y., Origami - Folded Plate Structures, Architecture Folding patterns, In $10^{\text {th }}$ World Conference on Timber Engineering, Myazaki, Japan, 2008.

[3] Lebée, A, From Folds To Structure, A Review, International Journal of Space Structures, 30, 2, 2015, 55-74.

[DOI: 10.1260/0266-3511.30.2.55]

[4] Tachi T., Freeform Rigid-Foldable Structure using Bidirectionally Flat-Foldable Planar quadrilateral Mesh. In Advances in Architectural Geometry 2010. Springer Vienna, 2010, 87-102.

[DOI: 10.1007/978-3-7091-0309-8_6]

[5] Robeller C., Weinand $\boldsymbol{Y}$., Interlocking folded plate - integral mechanical attachment for structural wood panels. Int. Journal of Space Structures, 30, 2, 2015, 111-122

[6] Schief W.K., Bobenko A., Hoffmann T., On the Integrability of Infinitesimal and Finite Deformations of Polyhedral Surfaces, Discrete Differential Geometry, Springer, 2008, 67-93.

[7] Harding J, Lewis H., The TRADA Pavilion - A Timber Plate Funicular Shell. In 2013 IASS Annual Symposium: Beyond the Limits of Man, 2013. 
[8] Li J.-M., Knipper J., Segmental timber plate shell for the Landesgartenschau exhibition Hall in Schwäbischgmünd - the application of fingerjoint in plate structure. International. Journal of Space Structures, 30, 2, 2015, 123-129.

[9] Robeller, C., Weinand $Y$., Fabrication-Aware Design of Timber Folded Plate Shells with Double Through Tenon Joints in Robotic Fabrication in Architecture 2016, p. 166177, 2016.

[10] Sticic A., Weinand Y., Timber Folded Plate Structures - Topological and Structural Considerations. International Journal of Space Structures, 30, 2, 2015, 169-178.

[11] Malek, S. R. The effect of geometry and topology on the mechanics of grid shells (Doctoral dissertation, Massachusetts Institute of Technology), 2012.

[12] Hahn B., Analyse und Beschreibung eines raümlichen Tragwerks aus Massivholzplatten., EPFL, Tech. rep., 2009.

[13] Falk A., Von Buelow P. Form Exploration of Folded Plate Timber Structures based on Performance Criteria. Full Papers: Taller, Longer, Lighter (2011).

[14] Von Buelow P., Falk A., Turrin M. Optimization of structural form using a genetic algorithm to search associative parametric geometry. Proc. International Conference Structures and Architecture 2010.

[DOI: 10.1201/b10428-93]

[15] Mesnil R., Douthe C., Baverel O., Leger B., Linear buckling of quadrangular and Kagome grids: a comparative assessment, Engineering Structures, 132, 2017, 337-348.

[DOI: 10.1016/j.engstruct.2016.11.039]

[16] Mesnil R. Douthe C., Baverel O., Leger B., Marionette mesh: from descriptive geometry to fabrication-aware design, In Advances in Architectural Geometry 2016, 2016.

[17] Preisinger $\boldsymbol{C}$., Linking Structure and Parametric Geometry, Architectural Design, 83, 2013, 110-113.

[DOI: 10.1002/ad.1564]

[18] Argyris, J. H., Papadrakakis, M., Apostolopoulou, C., \& Koutsourelakis, S. The TRIC shell element: theoretical and numerical investigation. Comp. Methods in Applied Mechanics and Engineering 2000, 182(1-2), 217-245.

[DOI: 10.1016/S0045-7825(99)00094-8]

[19] Bulenda, T., \& Knippers, J. Stability of grid shells. Computers \& Structures, 2001, 79(12), 1161-1174.

[DOI: 10.1016/S0045-7949(01)00011-6]

[20] Mesnil, R., Ochsendorf, J. \& Douthe, C. Stability of Pseudo-Funicular Elastic Grid Shells. Int Journal of Space Structures, 2015, 30(1), 27-36.

[DOI: 10.1260/0266-3511.30.1.27]

[21] Lefevre, B., Douthe, C., \& Baverel, O., Buckling of elastic gridshells. Journal IASS, 2015, 56(3), 153-171.

[22] Norman, A. D., Seffen, K. A., \& Guest, S. $D$., Morphing of curved corrugated shells. International Journal of Solids and Structures, 2009, 46(7), 1624-1633.

[DOI: 10.1016/j.ijsolstr.2008.12.009]

[23] Jiang, C., Tang, C., Vaxman, A., Wonka, P., \& Pottmann, H., Polyhedral patterns. ACM Transactions on Graphics (TOG), 2015; 34(6), 172.

[DOI: $10.1145 / 2816795.2818077]$

[24] Mesnil, R., Douthe C., Baverel O., Léger B., Möbius Geometry and cyclidic nets: A framework for complex shape generation in architecture, In Proc. IASS 2016, Amsterdam. 


\section{APPENDIX}

\section{Complementary results on closed domes}

The synthesis of the results on the behaviour of closed domes under non-symmetrical loads is presented in this appendix.

\section{AT Strategy}

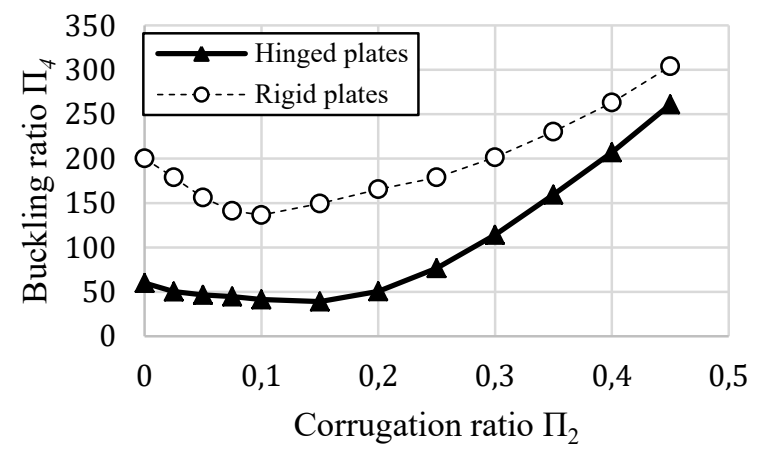

Figure 27: AT strategy, $t / L=1 \%, h / L=0.35$

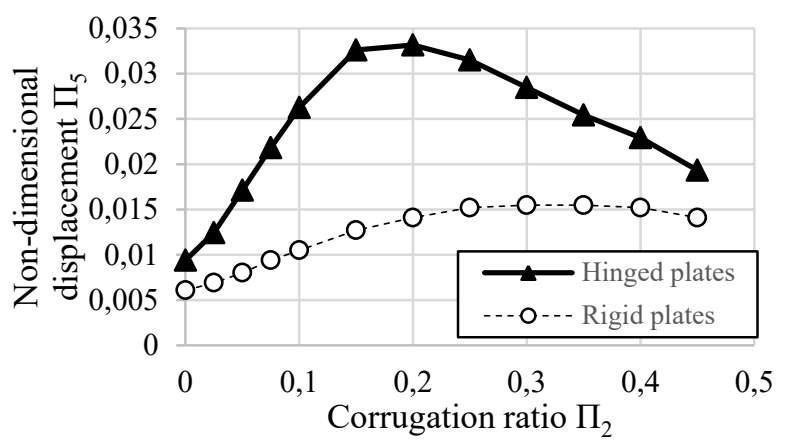

Figure 28: Non-dimensional displacement vs. corrugation ratio $($ AT strategy $, t / L=1 \%, h / L=0.35)$

\section{RT Strategy}

The curves show a pessimum occurring for moderate corrugation $\left(\Pi_{2}=0.15\right)$. Like for the shallow some, the corrugation does not improve the behaviour of the dome as much as the AT strategy.

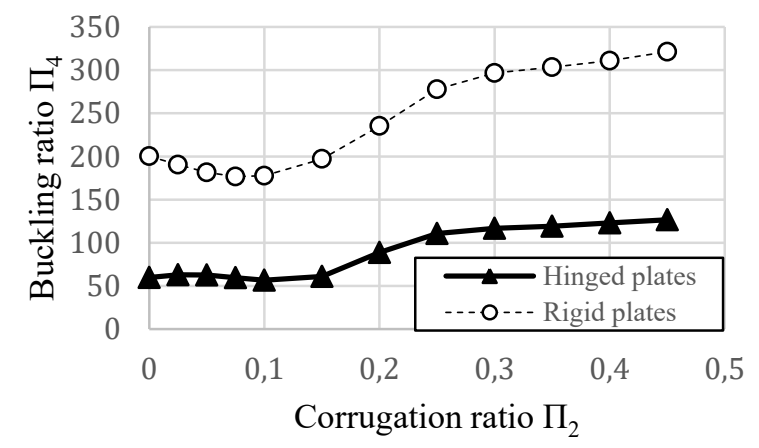

Figure 29: Buckling load vs. corrugation factor $(t / L=1 \%$, $h / L=0.35$, $R$ T strategy)
Again, the RT strategy leads to stiffer structures than the AT strategy for the closed domes, but the gain compared to the reference geometry are very limited, so that corrugation can be questioned when considering displacements only for closed domes.

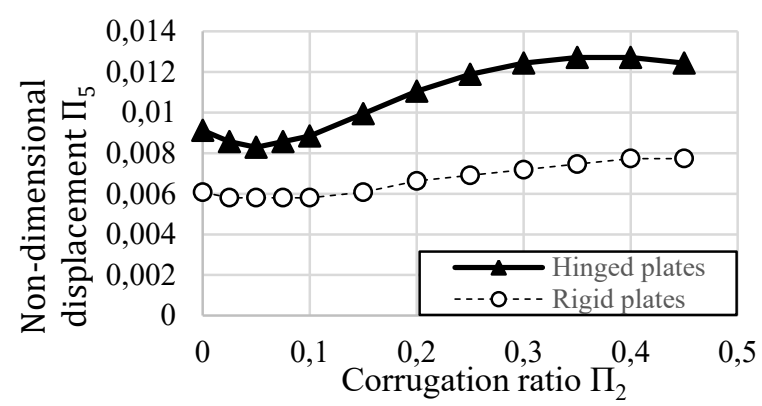

Figure 30: Non-dimensional displacements $(t / L=1 \%$, $h / L=0.35$, $R T$ strategy)

\section{Results on domes with free edge}

\section{AT strategy}

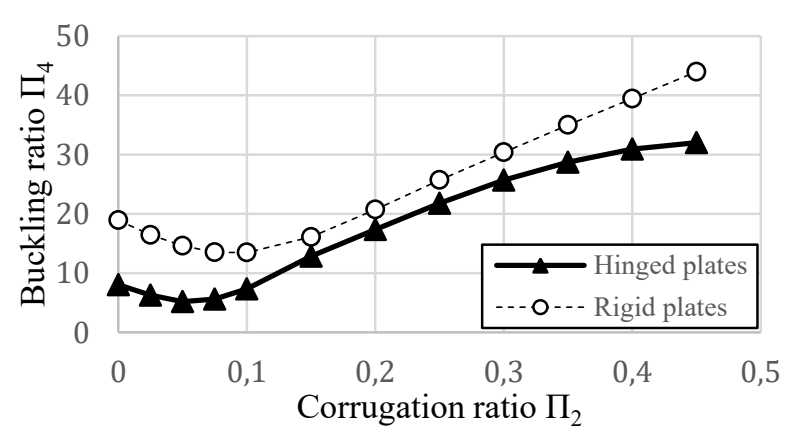

Figure 31: Buckling load vs. corrugation factor (AT strategy, $t / L=1 \%, h / L=0.35$ )

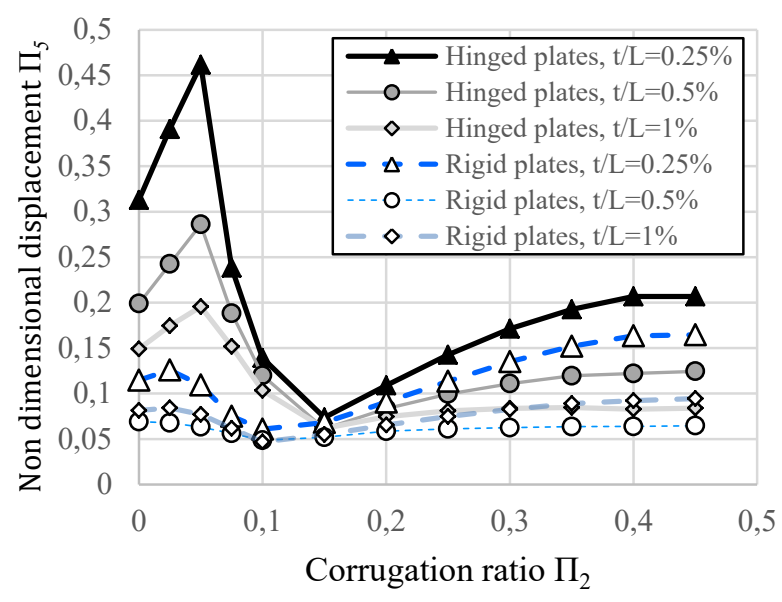

Figure 32: Non-dimensional displacements (AT strategy, $h / L=0.35$ ) 


\section{RT Strategy}

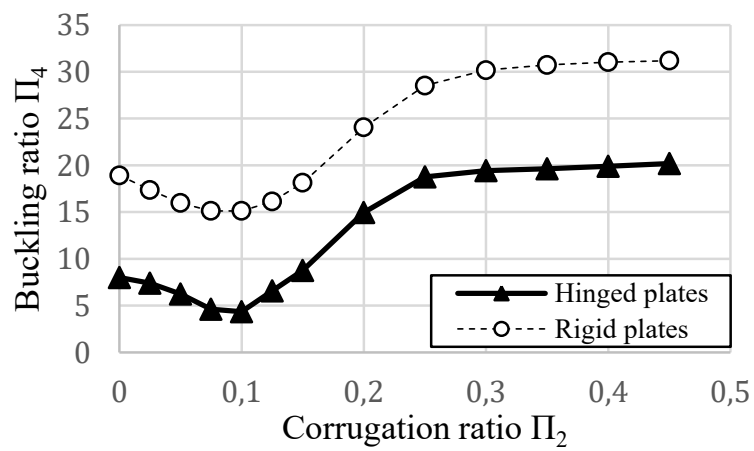

Figure 33: Buckling load vs. corrugation factor (RT strategy, $t / L=1 \%, h / L=0.35$ )

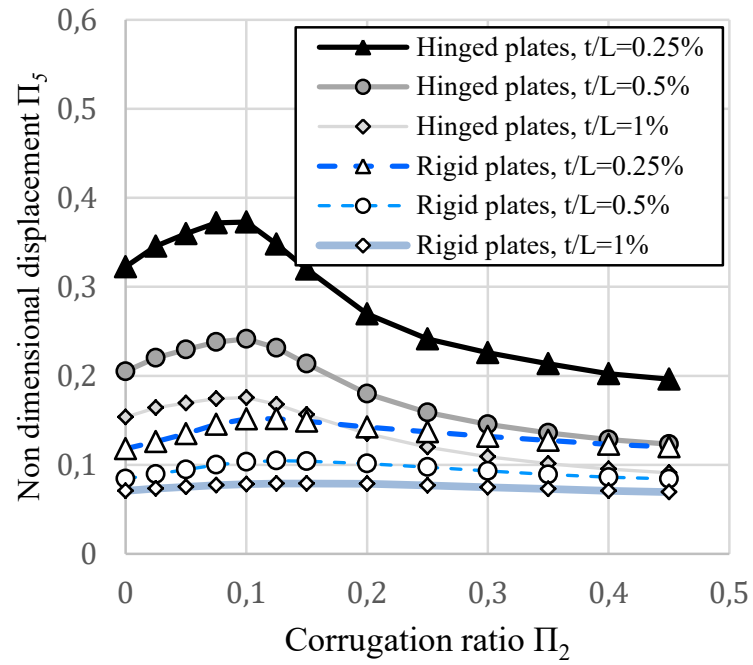

Figure 34: Non-dimensional displacements (RT strategy, $h / L=0.35$ ) 